\title{
Application of Rotational Isomeric State Theory to Ionic Polymer Stiffness Predictions
}

\author{
Lisa Mauck Weiland \\ Department of Mechanical Engineering, University of Pittsburgh, Pittsburgh, PA, 15261, lweiland@engr.pitt.edu \\ Emily K. Lada \\ Statistical and Applied Mathematical Sciences Institute (SAMSI), Research Triangle Park, NC, 27709-4006, \\ eklada@eos.ncsu.edu \\ Ralph C. Smith \\ Center for Research in Scientific Computation, Department of Mathematics, North Carolina State University, Raleigh, \\ NC, 27695, rsmith@eos.ncsu.edu \\ Donald J. Leo \\ Center for Intelligent Material Systems and Structures, Department of Mechanical Engineering, \\ Virginia Polytechnic Institute and State University, Blacksburg, VA, 24061, donleo@vt.edu
}

Presently, Rotational Isomeric State (RIS) theory directly addresses polymer chain conformation as it relates to mechanical response trends. The primary goal of this work is to explore the adaptation of this methodology to the prediction of material stiffness. This multi-scale modeling approach relies on ionomer chain conformation and polymer morphology and thus has potential as both a predictive modeling tool and a synthesis guide. The Mark-Curro Monte Carlo methodology is applied to generate a statistically valid number of end-to-end chain lengths via RIS theory for four solvated Nafion cases. For each case, a probability density function for chain length is estimated using various statistical techniques, including the classically applied cubic spline approach. It is found that the stiffness prediction is sensitive to the fitting strategy. The significance of various fitting strategies, as they relate to the physical structure of the polymer, are explored so that a method suitable for stiffness prediction may be identified.

\section{Introduction}

Ionic polymers comprise the active layer in Ionic Polymer-Metal Composites (IPMCs), which were first identified just over a decade ago [1-5] and now constitute an emerging class of soft transducers (see Figure 1). Because they generate large strain in response to low electric field stimulation and 


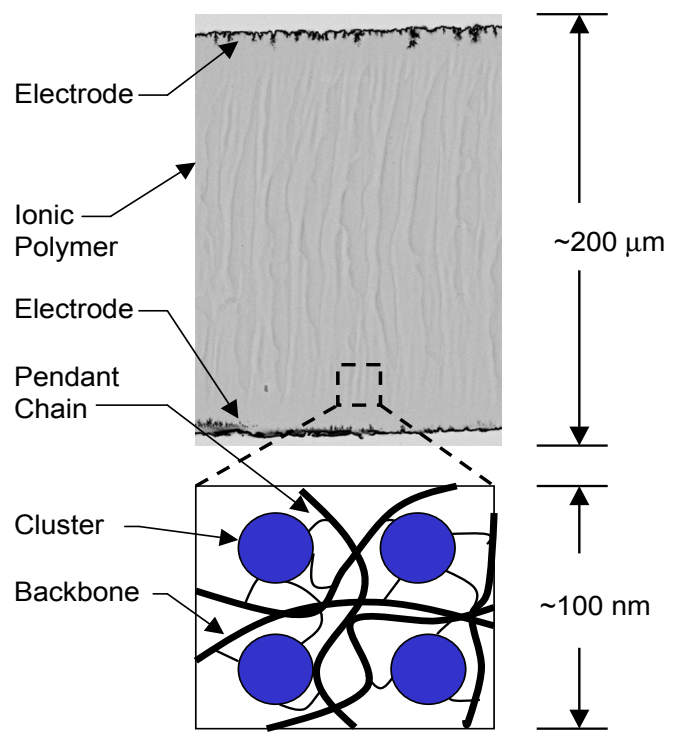

Figure 1: Cross-sectional view of a typical IPMC with illustration of the assumed ionic polymer clustering morphology based on Hsu and Geirke [12].

have high gravimetric energy density, there has been considerable conjecture over the potential applications of these soft transducers $[2,3,6-8]$ and considerable fruitful investigations towards these ends [9-11]. The high gravimetric energy density is a function of both the electromechanical transduction mechanisms of the ionic polymer layer and the global IPMC stiffness. Most models of the transduction behavior rely on the cluster morphology of the ionic polymer layer, as proposed by Hsu and Gierke [12], where the material has been solvated with water. In brief, the backbone of the ionic polymer chain is hydrophobic whereas the side chains terminate in hydrophilic ionic groups. Hsu and Geirke propose a clustering of these hydrophilic ionic side groups and the water that has been taken up by the material. The model further suggests an idealized structuring whereby the clusters are of essentially constant radius, uniformly distributed throughout the material, and interconnected by channels. Subsequently, many have sought to quantify the cluster radius and spacing as functions of polymer equivalent weight, hydration level, counterion, etc. — see for instance the modeling works of Datye and coworkers $[13,14]$ and the experimental work of Lehmani et al. [15] and Barbi et al. [16].

Current transduction models tend to be categorized in two classes: models assuming a hydraulic description of the electromechanical response $[17,18]$ and hybrid models including electrostatic, elastic, and osmotic effects $[8,19,20]$. Despite the disagreement over the underlying electromechanical 
mechanisms, the resulting predictions of all of the above modeling approaches are dependent upon ionomer stiffness. Moreover, it has been noted that the ionomer stiffness affects transducer response both directly, via classic energy density consideration, as well as indirectly, via an impact on transport properties [20,21]. Thus a modeling method which can predict ionomer stiffness as a function of its specific composition (ionomer type, counter ion type, solvation type and level, etc.) is desirable.

Existing stiffness prediction approaches fall short of this objective by being either too case specific or too general. For instance, as described by Treloar [22], the variation in rubber stiffness as a function of fluid uptake has long been estimated by

$$
G_{\mathrm{wet}}=G_{\mathrm{dry}} v^{1 / 3}
$$

where $G$ is the shear modulus and $v$ is the volume fraction of rubber in the rubber-liquid mix. Further, incompressibility is a classic assumption of rubber elasticity; thus Poisson's ratio is set to 0.5 and Young's modulus may also be directly solved. Unfortunately, experimental data indicates that while many aspects of ionomer mechanical behavior parallel that of rubber, the way in which solvent is taken up (by way of clustering) is fundamentally different than for a classic rubber, and hence the above general relation becomes invalid. A second, empirical approach is proposed by Li and Nemat-Nasser [19], based on the works of Hsu and Geirke [12] and Grot et al. [23]. For the specific case of Nafion, Young's modulus is given by

$$
E\left(c_{\mathrm{w}}\right)=E_{0} \exp \left[-\alpha\left(\frac{100 c_{\mathrm{w}} \rho_{\mathrm{w}}}{\left(1-c_{\mathrm{w}}\right) \rho_{\mathrm{d}}}+\frac{1200-\mathrm{EW}}{20}\right)\right]
$$

where $E_{0}=275 \mathrm{MPa}, \alpha=0.0294, c_{\mathrm{w}}$ is the water volume fraction, $\rho_{\mathrm{w}}$ and $\rho_{\mathrm{d}}$ are the densities of water and dry polymer, and EW is the equivalent weight of the Nafion sample in question. Example predictions for this empirical relation are $131 \mathrm{MPa}$ and $100 \mathrm{MPa}$ for fully hydrated (via boiling) Nafion 1100 equivalent weight with sodium and lithium as the counterions, respectively. When compared to the experimentally measured values of $80 \mathrm{MPa}$ and $75 \mathrm{MPa}$ respectively [8], this estimate is good for the current state of the art; however, it has been formulated specifically for Nafion rather than for ionomers in general. 
Rotational Isomeric State (RIS) theory as described by Flory [24] has long been used as a means to anticipate mechanical response trends in polymeric materials based on the underlying polymer chain conformation. The fundamental idea of this approach is that any given bond within a single polymer chain is limited to a discrete number of possible bond angles, based on corresponding low energy states. From this base, any number of statistical methodologies may be applied to anticipate final chain conformation and corresponding response to load. As discussed by Curro and Mark [25], application of the Gaussian approach to RIS theory, as described by Flory, does not represent a reasonable approximation for short chain polymers displaying rubber elasticity. A more realistic approach is to apply RIS theory in combination with a Monte Carlo methodology, from which a large number of end-to-end distances $r$ are generated. The $r$ values can then be used to estimate an appropriate probability density function $P(r)$ for the short chain case. A cubic spline approximate provides one technique that has been employed to estimate $P(r)$.

The Mark-Curro approach has also been applied to investigations on the effect of particle reinforcement [26-28]. In these particle reinforcement investigations, various volume fractions, inclusion sizes, and inclusion shapes and orientations are considered, where the chain conformation is excluded from occupying any volume dedicated to an inclusion. Thus, the predicted constitutive responses are due solely to the altered conformations of the polymer matrix and do not account for the load carrying capacity of the inclusions. A later preprint [29] suggests that efforts are underway to account for the load carrying capacity of the inclusions as well, and subsequently the methodology may be used for stiffness predictions. To date this method has not been used for stiffness predictions for reasons largely related to the limitations introduced by way of the simplifying assumptions (i.e., the commonly applied 3-state models of chain bond angles are unable to capture helical chain coiling [30]; uncompensated free-end effects, accurate identification of polymer molecular weight, etc. $[22])$.

In this paper it is hypothesized that reasonable estimations for the above noted limitations are possible so long as a more appropriate statistical approach is first employed. Stiffness predictions are thus considered for various similar, solvated ionomers.

It is understood that ionomer stiffness is a function of multiple parameters including ionomer type, solvation type and level, and counterion type. In this work RIS theory is applied in a manner 
analogous to the particle inclusion works [26-28] in order to capture each of these contributions, where inclusion volumes are now taken to be clusters. Cluster morphology is assumed a priori, based on available experimental measurements for the various cases; the subsequent backbone conformation in response to this morphology is applied to obtain stiffness predictions. In addition, it is assumed that these clusters, under specified conditions, may act as backbone cross-linking junctions, and thus the ionomer stiffness is based on polymer short chain response. Moreover, this computational approach permits direct elimination of free-end effects and provides a means to estimate a representative cluster-to-cluster molecular weight (or effective molecular weight based on the distance between cluster communication points on the polymer backbone).

The significant differences between the particle reinforcement investigations and the current technique are the following: (i) the inclusions are hydrophilic clusters with which the hydrophobic backbone may interact via the pendant chains, thus affecting the end-to-end distances considered, (ii) the probability density function $P(r)$ is estimated using various statistical approaches, and (iii) the various predictions are considered both in terms of the predicted trends and the magnitude of the predicted stiffness. It is found that stiffness predictions are sensitive to the choice of statistical methodology and thus represent a tool for expanding the capabilities of this modeling approach to stiffness predictions. Details of the various statistical approaches are discussed in [31]. In this work, the focus is on the impact these various methodologies have on the predicted response.

The paper is organized as follows. The second section discusses the RIS model construction for the ionic polymer Nafion in four hydrated forms: 1100 Equivalent Weight, in $\mathrm{Na}^{+}$and $\mathrm{Li}^{+}$forms, with two assumed cluster distributions each. Section three briefly describes the statistical analysis approaches and the fourth section presents model predictions and discussion.

\section{Multiscale Model Development}

The mechanical response of two hydrated Nafion 1100 Equivalent Weight cases in salt form are considered, one with $\mathrm{Na}^{+}$and the other with $\mathrm{Li}^{+}$counterions, where it is assumed that the ion exchange is complete. The Mark-Curro approach to RIS theory [25] is applied in a manner analogous to that employed in investigations focused on on the effect of particle reinforcement [26-28]. In the 


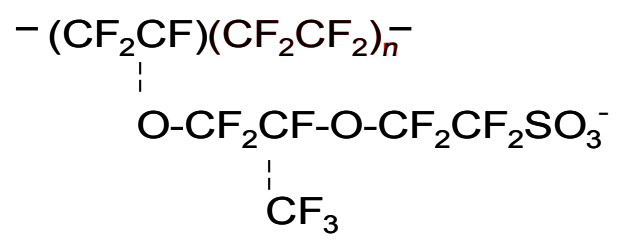

Figure 2: Chemical composition of a Nafion monomer.

particle reinforcement studies the chain conformation is excluded from occupying any volume dedicated to a rigid inclusion. In this investigation the inclusions take the form of hydrophilic clusters rather than rigid particles. The significant difference in the nature of the inclusion is addressed by way of the additional constraint that the backbone may have discrete points of communication with the hydrophilic clusters via the pendant chain attachment points. These attachment points are assumed to approximate cross-linking points, and thus mark an end-point for the given chain length $r$.

\subsection{Simulation Model for Estimating Chain Length}

To implement this model, appropriate statistical weight matrices for backbone chain conformation, bond lengths, and distances between pendant chain attachment points must be identified. The repeating monomer unit in the chemical structure of Nafion is depicted in Figure 2, where $\left(\mathrm{CF}_{2} \mathrm{CF}\right)\left(\mathrm{CF}_{2} \mathrm{CF}_{2}\right)_{n}$ represents the repeating unit of the backbone and $\mathrm{SO}_{3}^{-}$is the pendant chain terminal ionic group which tends toward the hydrophilic clusters. If the 87/13 Nafion case is considered, meaning that there are approximately $13\left(\mathrm{CF}_{2} \mathrm{CF}\right)$ groups to every $87\left(\mathrm{CF}_{2} \mathrm{CF}_{2}\right)$ groups in the total length of the backbone, then $n$ is most often approximately 7 . In other words, the bulk of the backbone takes the form of PTFE, or Teflon $[32,33]$. Because it is the backbone of the polymer which is responsible for the load carrying capacity of the ionomer, the PTFE conformation is assumed for all bond placements except pendant chain attachment points.

Whereas a typical value for $n$ in the chemical structure of Nafion is approximately 7 , there is in fact variability in this value. To account for this variation, $n$ is sampled from a discrete probability distribution with a minimum of 5 , a maximum of 11 , and a mean of approximately 7 [31].

Still to be addressed is the number of times the repeat unit of Figure 2 is expected for a single polymer strand of Nafion. Because the actual molecular weight is not readily available, 


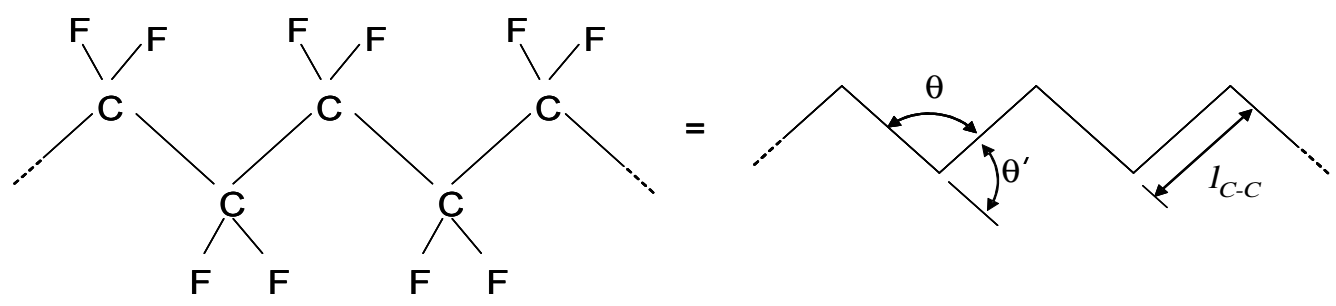

Figure 3: Depiction of the in-plane bond angle $\theta=116^{\circ}$ and PTFE backbone component length $l_{\mathrm{C}-\mathrm{C}}=1.53 \AA$. The angle $\theta^{\prime}$ is the supplementary angle to $\theta$ having the value $64^{\circ}$.

in part because polymers do not have fixed chain lengths, a distribution of lengths is assumed. The assumed distribution anticipates that total chain lengths contain between 135 and 225 repeat units. Note, however, that the lack of available information on this parameter is considered noncritical because this material's load bearing capability is based on the distance between cluster communication points along a given backbone rather than on the entire backbone chain length.

The model progression begins with the placement of a cluster configuration in a 3-dimensional grid of $(5000 \AA)^{3}$. The grid size is selected to accommodate the longest anticipated, fully extended backbone. Two cluster distribution schemes are considered as detailed in the preliminary work [31]. The first relies directly on the simplified model of Hsu and Gierke [12] where the clusters are uniformly distributed on a square grid analogous to the cubic crystalline structure. The second uniformly distributes clusters of the same radius and net volume fraction as the first case, but on a staggered grid analogous to the HCP crystalline structure. The cluster size and volume fraction for the cases studied are provided in Table 1. For simplicity, calculation of the volume taken up by the ionic groups is currently neglected. Should this prove significant, the estimation proposed by Hsu and Gierke [12] can be applied to cluster redistribution.

Once the cluster distribution is assigned, a single PTFE bond is randomly placed in the 3-

Table 1: Cluster Morphology Values based on [8].

\begin{tabular}{ccc}
\hline \hline & $\mathrm{Li}^{+}$ & $\mathrm{Na}^{+}$ \\
\hline Cluster Volume Fraction & $38 \%$ & $30 \%$ \\
Cluster Radius & $23 \AA$ & $21 \AA$ \\
Cluster Center-to-Center Separation & $50.6 \AA$ & $50.4 \AA$ \\
\hline
\end{tabular}


dimensional grid, where cluster locations are excluded. For PTFE the in-plane bond angle and length are fixed at $\theta=116^{\circ}$, and $l_{\mathrm{C}-\mathrm{C}}=1.53 \AA$, respectively, as illustrated in Figure 3 . Thus, the in-plane angle of the second bond is known; however, a statistical weight matrix must be applied in order to identify an appropriate out-of-plane rotation angle, where it is understood that various angular orientations between bonds correspond with local low energy conformations. Each subsequent bond must be similarly placed. For PTFE, both 3-state and 4-state statistical weight matrices have previously been studied, where the 4-state matrices have been shown to be somewhat preferred [30]. For the 4 -state case the possible out-of-plane angles are $+15^{\circ},+120^{\circ},-120^{\circ}$, and $-15^{\circ}$ (or trans + , gauche+, gauche-,trans-). The 4-state matrices applied are

$$
\begin{gathered}
U_{2}=\left[\begin{array}{cccc}
1 & 0 & 0 & 0 \\
0 & \sigma^{\prime} & 0 & 0 \\
0 & 0 & \sigma^{\prime} & 0 \\
0 & 0 & 0 & 1
\end{array}\right], \quad U_{3}=\left[\begin{array}{cccc}
1 & \sigma & 0 & \omega \\
1 & \sigma & \sigma \beta & 1 \\
1 & \sigma \beta & \sigma & 1 \\
\omega & 0 & \sigma & 1
\end{array}\right], \\
U_{k}=\left[\begin{array}{llll}
1 & \sigma & 0 & \omega \\
1 & \sigma & 0 & 0 \\
0 & 0 & \sigma & 1 \\
\omega & 0 & \sigma & 1
\end{array}\right], \quad U_{N}=\left[\begin{array}{ccccc}
1 & \sigma^{\prime} & \sigma^{\prime} & \omega \\
1 & \sigma^{\prime} & \sigma^{\prime} \beta & 0 \\
0 & \sigma^{\prime} \beta & \sigma & 1 \\
\omega & \sigma & \sigma^{\prime} & 1
\end{array}\right],
\end{gathered}
$$

where $\sigma=0.2, \sigma^{\prime}=2.0, \omega=0.2, \beta=0.5$ at $25^{\circ} \mathrm{C}[30]$, and the rows and columns are indexed in the order $+15^{\circ},+120^{\circ},-120^{\circ}$, and $-15^{\circ}$. After these matrices have been normalized [31], the orientation of the second bond is dictated by $U_{2}$, the third by $U_{3}$, the last by $U_{N}$, and all others by $U_{k}$, with two exceptions. The matrices are overridden when a bond placement coincides with a cluster or when the bond corresponding to a pendant chain attachment point is being placed. The first exception addresses the reality that the backbone chain is hydrophobic and will reconfigure upon solvation to an alternate low energy state in order to avoid the hydrophilic region. The second exception addresses the reality that the terminal ionic group will tend toward a hydrophilic region and again locally reconfigure the chain upon solvation. It is understood that application of the 


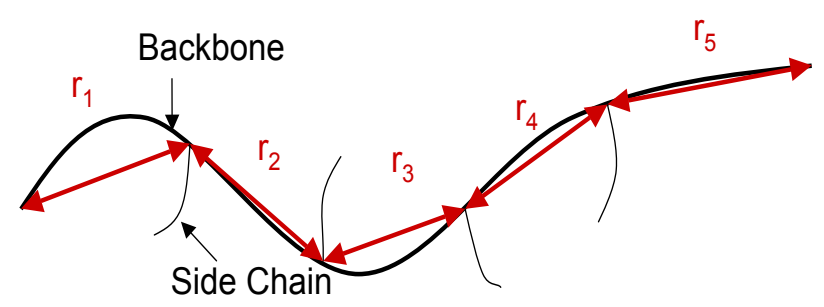

Figure 4: Distance $r$ between cluster interaction points for a single Nafion polymer chain.

in-plane PTFE bond angle in this case is a simplifying assumption.

When a bond placement would locate the next bond within a cluster, another random number is generated to find an alternate but viable low energy conformation. If all out-of-plane rotation angles result in cluster coincidence, the chain is terminated. When a bond corresponding to a pendant chain connection point is being placed, of the possible $\pm 15^{\circ}$ and $\pm 120^{\circ}$ out-of-plane PTFE rotations, the angle that minimizes the distance between the carbon atom with the attached side chain and the nearest cluster is used. It is assumed that the pendant chain terminal ionic group successfully communicates with the nearest cluster if the distance between the backbone connection point and the nearest cluster is within $8 \AA$. This value is an estimate of the fully extended length of the pendant chain. It is found that application of this value leads to between $75 \%$ and $92 \%$ successful cluster communication depending on the assumed cluster configuration. It is not expected that $100 \%$ of the ionic groups will reside within a cluster and thus, this range is deemed reasonable.

Each simulated Nafion backbone chain can have multiple $r$ values as illustrated in Figure 4. Furthermore, the first and last $r$ value for each chain, or free ends, do not support any load, and therefore do not contribute to stiffness. Predictions with and without correction of the free-end effects are considered.

In order to assure statistical validity, a large number of $r$ values (about 10,000) are generated. From this, an expression for the chain length probability density function $P(r)$ must be estimated. Development of an appropriate expression is the subject of Section 3.

\subsection{Macroscopic Model for Estimating Stiffness}

Assuming an appropriate $P(r)$ expression has been developed, per Boltzmann's approach to statistical thermodynamics, it can be directly related to the entropy of the chain as a function of the 
number of conformations available for a given state [25],

$$
S(r)=c+k \ln P(r)
$$

where $k$ is Boltzmann's constant and $c$ is a constant of integration which drops out when the difference in entropy for the unperturbed configuration is taken with respect to the distorted configuration. In this approach it is assumed that under load the rotation about the individual bonds is unrestricted which allows the assumption that the Helmholz free energy is strictly a function of entropy. For the assumptions of rubberlike elasticity, the "three chain" model, as described by Treloar [32], yields the relation

$$
\Delta S=\frac{\nu}{3}\left[S\left(r_{\mathrm{o}} \alpha\right)+2 S\left(r_{\mathrm{o}} \alpha^{-1 / 2}\right)-3 S\left(r_{\mathrm{o}}\right)\right]
$$

for the change in entropy upon distortion. Here $\nu$ is the number density of network chains, $r_{\mathrm{o}}$ is the root mean square of the $r$ values applied in the development of $P(r)$, and $\alpha=L / L_{i}$ is the relative length of the sample. The nominal stress $f^{*}$ is then given by

$$
f^{*}=-T\left(\frac{\partial \Delta S}{\partial \alpha}\right)_{T}=-\frac{\nu k T r_{\mathrm{o}}}{3}\left[G^{\prime}\left(r_{\mathrm{o}} \alpha\right)-\alpha^{-3 / 2} G^{\prime}\left(r_{\mathrm{o}} \alpha^{-1 / 2}\right)\right]
$$

where $G(r)=\ln P(r)$ and $G^{\prime}(r)=\frac{d G(r)}{d r}$. The corresponding modulus $\left[f^{*}\right]$ is given as

$$
\left[f^{*}\right]=\frac{f^{*}}{\alpha-\alpha^{-2}}
$$

For small strains $(\alpha \rightarrow 1)$, the modulus in (7) approaches Young's modulus for the polymer matrix alone; this small strain modulus will be referred to as $E_{\text {init }}$. The global Youngs's modulus, $E_{\text {ave }}$, is expected to be lower than $E_{\text {init }}$ due to the volume fraction of hydrophilic clusters which are not expected to be load bearing. Because only the global stiffness, $E_{\text {ave }}$, is experimentally available the means to properly express $E_{\text {init }}$, and subsequently relate it to $E_{\text {ave }}$ are required. Consider first the expression for $E_{\text {init }}$. 
Substituting equation (6) into equation (7) and applying L'Hôpital's rule, yields the relation

$$
E_{\text {init }}=-\frac{\nu k T r_{\mathrm{o}}}{6}\left\{\frac{r_{\mathrm{o}} P\left(r_{\mathrm{o}}\right) P^{\prime \prime}\left(r_{\mathrm{o}}\right)-r_{\mathrm{o}}\left[P^{\prime}\left(r_{\mathrm{o}}\right)\right]^{2}+P\left(r_{\mathrm{o}}\right) P^{\prime}\left(r_{\mathrm{o}}\right)}{\left[P\left(r_{\mathrm{o}}\right)\right]^{2}}\right\}
$$

for the initial stiffness.

In order to relate $E_{\text {init }}$ to $E_{\text {ave }}$, it is first noted that $E_{\text {init }}$ is directly analogous to matrix stiffness, $E_{m}$, of classic composite materials theory. Thus, a modified Voigt-Reuss approximation for plane stress [34] is used to relate $E_{\text {init }}$ to the experimentally available values, $E_{\text {ave }}$. This approximation is given by

$$
E_{\text {init }}=E_{m}=\frac{1}{f} E_{\text {ave }}=V_{p} E_{\text {ave }}
$$

where $f$ is the volume fraction not taken up by water, which can be equivalently expressed in terms of the normalized volume after uptake $V_{p}$ (i.e., if upon hydration the volume increases by $44 \%$, then $V_{p}$ is 1.44$)$.

Traditionally RIS has been used for the purpose of studying mechanical response trends where model predictions described by equations (6) and (7) are normalized with respect to $v k T$. However, a value must be assigned to this parameter in order to predict stiffness. Identification of the number density of chains $\nu$ requires the application of some simplifying assumptions. To begin, $\nu$ is given by

$$
\nu=\frac{\rho N_{\mathrm{A}}}{\mathrm{MW}_{\mathrm{c}-\mathrm{c}}}
$$

where $\rho$ is the material density, $N_{\mathrm{A}}$ is Avagadro's number, and $\mathrm{MW}_{\mathrm{c}-\mathrm{c}}$ is the molecular weight of the portion of the chain between two cluster communication points.

The material density is a readily available, experimentally determined value, whereas Avagadro's number is a constant. This leaves $\mathrm{MW}_{\mathrm{c}-\mathrm{c}}$, which is estimated based on the percentage of successful pendant chain-to-cluster communications. The estimate assumes that $n=7$ in the bulk of the repeat units (Figure 2), which corresponds to a molecular weight of that repeat unit of $781 \mathrm{~g} / \mathrm{mol}$, where again, only the backbone is considered to be load bearing. It is next assumed that failed 
cluster communications are sufficiently infrequent that it is highly improbable that there would be two failures in sequence. Thus, a single failure corresponds to a local $\mathrm{MW}_{\mathrm{c}-\mathrm{c}}$ of $1562 \mathrm{~g} / \mathrm{mol}$. Applying these values as a ratio of their occurrence is then used to estimate $\mathrm{MW}_{\mathrm{c}-\mathrm{c}}$. For example, if the failure rate is $15 \%$, then $\mathrm{MW}_{\mathrm{c}-\mathrm{c}} \approx 0.85(781)+0.15(1562)=898 \mathrm{~g} / \mathrm{mol}$.

\section{Statistical Analysis}

The details of the statistical fitting methods applied to the development of various $P(r)$ expressions for the same distribution of $r$ values are discussed in [31]. Provided here is a brief overview of the cubic spline and Johnson family methods. In the traditional approach of RIS Monte Carlo theory, a cubic spline approach is used to estimate $P(r)$. The simulation-generated $r$ values are divided into groups, or bins, and a knot value is specified for each bin so that on each interval (delimited by the knots), a cubic polynomial is fit to the data. If we define the indicator function $L_{i}(r)$ for the subinterval $\left(k_{i}, k_{i+1}\right]$, where $k_{i}$ is the $i$ th knot, as

$$
L_{i}(r)= \begin{cases}1, & \text { for } k_{i}<r \leq k_{i+1} \\ 0, & \text { otherwise }\end{cases}
$$

then the result is a piecewise-polynomial estimate of $P(r)$ of the form

$$
P(r)=\frac{1}{K} \sum_{i=1}^{n^{*}} L_{i}(r)\left[A_{i}\left(r-k_{i}\right)^{3}+B_{i}\left(r-k_{i}\right)^{2}+C_{i}\left(r-k_{i}\right)+D_{i}\right]
$$

where $n^{*}$ is the number of knots, $A, B, C$, and $D$ are the corresponding coefficients of the cubic equations, and $K$ is a normalizing constant so that the function $P(r)$ given in (12) integrates to unity. While this expression is easily differentiable for substitution into equations (4)-(7), one challenge with this approach is that cubic polynomials are often not sufficiently flexible to accurately model the tail behavior of the density. Additionally, the shape of the estimated density is highly dependent on the number and location of the knots. For example, if the number of knots is large, the corresponding estimate of $P(r)$ not only gives the visual impression of being unnaturally noisy, but also yields nonphysical response trends. Finally, the details of the fitting methodology (such as knot selection) are typically not well-defined. In the current work, a series of fits are considered 
where the number and location of knots is varied, in order to illustrate the physical implications of using a cubic spline approach.

Alternatively, the application of the Johnson family of distributions for density estimation is clearly defined, effectively eliminating any ambiguity in defining the fitting procedure. Further, unlike the coefficients in the cubic spline approach, the various parameters of the Johnson distribution have specific statistical meaning. Lastly, the Johnson distribution only requires the estimation of four parameters and the resulting expression can be easily applied in equations (4)-(8).

In general, the probability density function $P(r)$ based on the Johnson distributions has the form

$$
P(r)=\frac{\delta}{\lambda \sqrt{2 \pi}} f^{\prime}\left(\frac{r-\xi}{\lambda}\right) \exp \left\{-\frac{1}{2}\left[\gamma+\delta f\left(\frac{r-\xi}{\lambda}\right)\right]^{2}\right\}
$$

where $\delta$ and $\gamma$ are shape parameters, $\lambda$ is a scale parameter, $\xi$ is a location parameter, the function $f(\cdot)$ is defined according to the applied distribution family, and $f^{\prime}(\cdot)$ is the first derivative of $f(\cdot)$ with respect to $r[31,35]$. The distribution families defining $f(\cdot), f^{\prime}(\cdot)$, and $f^{\prime \prime}(\cdot)$ are

$$
\begin{aligned}
& f(y)= \begin{cases}\ln (y), & \text { for the } S_{L} \text { (lognormal) family } \\
\ln \left(y+\sqrt{y^{2}+1}\right), & \text { for the } S_{U} \text { (unbounded) family, } \\
\ln \left(\frac{y}{1-y}\right), & \text { for the } S_{B} \text { (bounded) family } \\
y, & \text { for the } S_{N} \text { (normal) family; }\end{cases} \\
& f^{\prime}(y) \equiv \frac{d f(y)}{d y}=\left\{\begin{array}{cc}
1 / \sqrt{y^{2}+1,}, & \text { for the } S_{U} \text { (unbounded) family and all real } y, \\
1 /[y(1-y)], & \text { for the } S_{B} \text { (bounded) family and } y \in(0,1) \\
1, & \text { for the } S_{N} \text { (normal) family and all real } y ;
\end{array}\right.
\end{aligned}
$$




$$
f^{\prime \prime}(y) \equiv \frac{d^{2} f(y)}{d y^{2}}=\left\{\begin{array}{cl}
-1 / y^{2}, & \text { for the } S_{L} \text { family and } y>0 \\
-y /\left(y^{2}+1\right)^{3 / 2}, & \text { for the } S_{U} \text { family and all real } y \\
-(1-2 y) /[y(1-y)]^{2}, & \text { for the } S_{B} \text { family and } y \in(0,1), \\
0, & \text { for the } S_{N} \text { family and all real } y
\end{array}\right.
$$

The bounds on these distributions are

$$
\mathbf{H}= \begin{cases}{[\xi,+\infty)} & \text { for the } S_{L} \text { (lognormal) family } \\ (-\infty,+\infty) & \text { for the } S_{U} \text { (unbounded) family } \\ {[\xi, \xi+\lambda]} & \text { for the } S_{B} \text { (bounded) family } \\ (-\infty,+\infty) & \text { for the } S_{N} \text { (normal) family }\end{cases}
$$

Using equations (13)-(17), the function $G(r)$ can be written as

$$
G^{\prime}(r)=\frac{f^{\prime \prime}\left(\frac{r-\xi}{\lambda}\right)-\delta\left[f^{\prime}\left(\frac{r-\xi}{\lambda}\right)\right]^{2}\left[\gamma+\delta f\left(\frac{r-\xi}{\lambda}\right)\right]}{\lambda f^{\prime}\left(\frac{r-\xi}{\lambda}\right)} .
$$

When this expression is substituted into equation (7), the resulting stiffness expression as a function of distortion is given by

$$
\begin{gathered}
{\left[f^{*}\right]=-\frac{r_{\mathrm{o}} \nu k T}{3\left(\alpha-\alpha^{-2}\right)}\left\{\frac{f^{\prime \prime}\left(\frac{r_{\mathrm{o}} \alpha-\xi}{\lambda}\right)-\delta\left[f^{\prime}\left(\frac{r_{\mathrm{o}} \alpha-\xi}{\lambda}\right)\right]^{2}\left[\gamma+\delta f\left(\frac{r_{\mathrm{o}} \alpha-\xi}{\lambda}\right)\right]}{\lambda f^{\prime}\left(\frac{r_{\mathrm{o}} \alpha-\xi}{\lambda}\right)}\right.} \\
\left.-\alpha^{-3 / 2} \frac{f^{\prime \prime}\left(\frac{r_{\mathrm{o}} \alpha^{-1 / 2}-\xi}{\lambda}\right)-\delta\left[f^{\prime}\left(\frac{r_{\mathrm{o}} \alpha^{-1 / 2}-\xi}{\lambda}\right)\right]^{2}\left[\gamma+\delta f\left(\frac{r_{\mathrm{o}} \alpha^{-1 / 2}-\xi}{\lambda}\right)\right]}{\lambda f^{\prime}\left(\frac{r_{\mathrm{o}} \alpha^{-1 / 2}-\xi}{\lambda}\right)}\right\} .
\end{gathered}
$$

Of the four Johnson family distributions initially considered (unbounded, bounded, lognormal, and normal), it is found that the lognormal and normal families are not sufficiently flexible for the data generated from the simulation model and both yield poor statistical fits. Subsequently, only the unbounded and bounded families are considered in detail. 


\section{Predictions and Discussion}

While a total of 135 to 225 repeat units (Figure 2) per ionomer chain is identified as the target, the chain length is typically terminated much earlier due to chain collision with a cluster. Actual simulated chain lengths $m$ never exceeded 45 . While early termination clearly leads to non-physical predictions of total chain length, it is not believed that this early termination presents a significant problem in the development of an appropriate $P(r)$ expression because it is the lengths between cluster interactions which dictate the elastic properties of the material. To ensure this, analyses have been performed where all chain lengths are included (including those which terminated early) as well as for the more physical case where all initial and final lengths are discarded to eliminate endeffects. Thus, in the current study, correction of free-end effects not only addresses the reality that free ends do not contribute to material stiffness, but also necessarily eliminates early termination effects. (For discussion of free end effects, see for instance, Treloar [22].)

Figures 5 and 6 demonstrate the effect of deleting the first and last $r$ value for each chain. From these two figures, it is evident that removing the end effects for each simulated backbone chain greatly improves the behavior of the data in the lower tail. Those $r$ values computed from chains with only one or two bonds have been eliminated and, as a result, the second mode in the lower tail of the density in Figure 5 is not as prominent in the density shown in Figure 6. Consequently, correction of free-end effects has the added advantage of greatly improving the accuracy of the Johnson fit since in general the Johnson distribution is not sufficiently flexible to quantify bimodal behavior.

\section{Predicted Stiffness Values}

Applying Equation (9), the matrix stiffness $\left(E_{\text {init }}=E_{m}\right)$ is estimated to be $120 \mathrm{MPa}$ and 115 $\mathrm{MPa}$ for $\mathrm{Li}^{+}$and $\mathrm{Na}^{+}$, respectively, where these values are based on the experimentally measured stiffness values $\left(E_{\text {ave }}\right)$ of $75 \mathrm{MPa}$ and $80 \mathrm{MPa}$ for the $\mathrm{Li}^{+}$and $\mathrm{Na}^{+}$cases [8]. Figures $7-14$ illustrate the predicted probability density functions, with the location of $r_{\mathrm{o}}$ indicated; corresponding stiffness

values for the various cases are provided in Tables 2 and 3. The cubic spline curve of Figure 7 illustrates the impact of uncorrected end effects in the current study via the second mode at very 


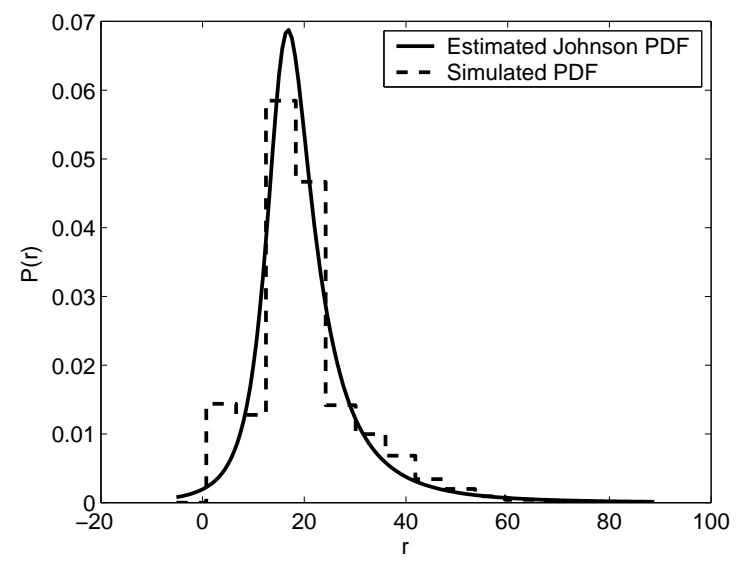

(a)

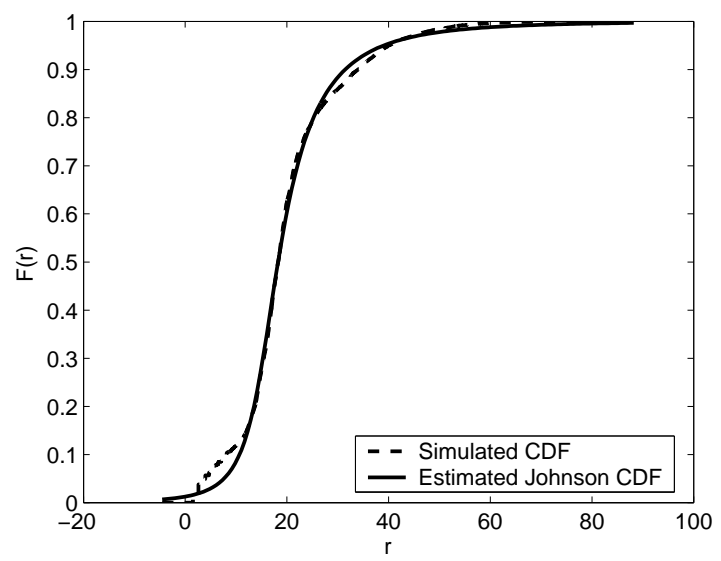

(b)

Figure 5: Estimated PDF (a) and CDF (b) for the sodium/rectangular case using a Johnson unbounded density function.

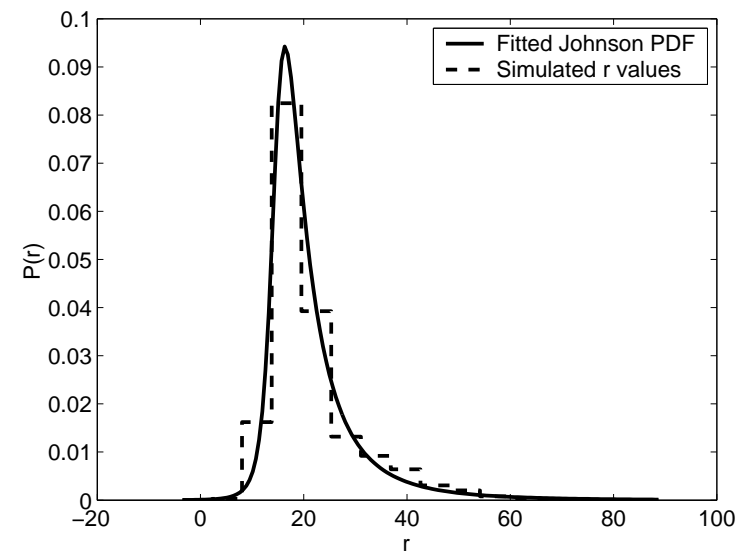

(a)

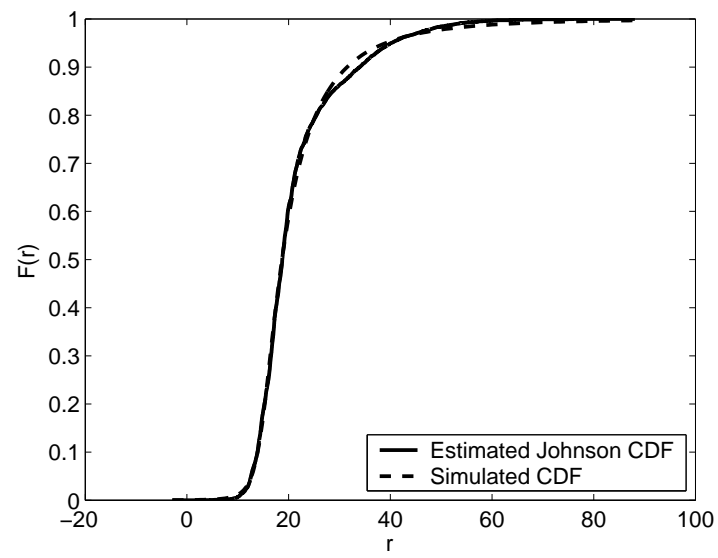

(b)

Figure 6: Estimated PDF (a) and CDF (b) for the sodium/rectangular case using a Johnson unbounded density function with corrected end effects.

short chain lengths. Per the discussion of the previous section, there is no physical reason, other than uncorrected end-effects, to justify the existence of this second mode as reasonable. Therefore, because of the aforementioned difficulties associated with fitting the cubic spline case even to reliable distribution data, no further cubic spline fits were employed for the nonphysical cases (uncorrected end-effects).

Because we expect all considered cases to have similar predicted stiffness values (120 MPa and $115 \mathrm{MPa}$ for $\mathrm{Li}^{+}$and $\mathrm{Na}^{+}$respectively), the bounded Johnson distribution is deemed favorable. This is because (a) all stiffness predictions for the Johnson bounded case are within a reasonable 
Table 2: Initial stiffness predictions [MPa] per statistical methodology, end effects neglected.

\begin{tabular}{cccc}
\hline \hline Cation/Grid Orientation & Cubic Spline & Johnson Unbounded & Johnson Bounded \\
\hline Sodium, Cubic & 3.5 & 3.2 & 6.4 \\
Sodium, HCP & & 3.4 & 9.8 \\
Lithium, Cubic & & -1.0 & 10.4 \\
Lithium, HCP & & 37.8 & 9.5 \\
\hline
\end{tabular}

Table 3: Initial stiffness predictions [MPa] per statistical methodology, corrected end effects.

\begin{tabular}{cccc}
\hline \hline Cation/Grid Orientation & Cubic Spline & Johnson Unbounded & Johnson Bounded \\
\hline Sodium, Cubic & -6.6 & 1.0 & 9.8 \\
Sodium, HCP & 26.9 & 9.1 & 13.8 \\
Lithium, Cubic & 27.8 & 4.0 & 13.6 \\
Lithium, HCP & 25.6 & 18.1 & 18.3 \\
\hline
\end{tabular}

range (standard deviation of 3.6 $\mathrm{MPa}$ from the mean of $10.0 \mathrm{MPa}$ ), while the others have much more scatter (standard deviation of $12.9 \mathrm{MPa}$ from the mean of 3.7 MPa for the Johnson unbounded case and standard deviation of $15.9 \mathrm{MPa}$ from the mean of 25.6 MPa for the cubic spline case); (b) the previously noted trend for sodium being marginally softer than lithium is most closely observed in the Johnson bounded case; and (c) the Johnson bounded yielded no negative stiffness predictions.

Because stable/repeatable results are of utmost importance in the development of a new predictive methodology, it is the stability of the predictions by the Johnson bounded case that is emphasized rather than the magnitude of the predictions themselves. Sources of error potentially responsible for the predicted low magnitude in stiffness include (a) the semi-crystalline nature of the polymer matrix, which is currently neglected; (b) while Figures 5 and 6 suggest that early termination introduces an nonphysical bias toward a second mode at very short chain lengths, and hence, correction of end-effects also corrects early termination effects, it is conceivable that early chain termination interferes with the prediction of longer chain lengths; (c) the use of a rough weight average estimate in the molecular weight calculation methodology (a more appropriate solution will require a statistical analysis of this distribution as well); and (d) the assumption of spherical cluster shapes, where oblate or rod-like clusters may be more physically accurate. 


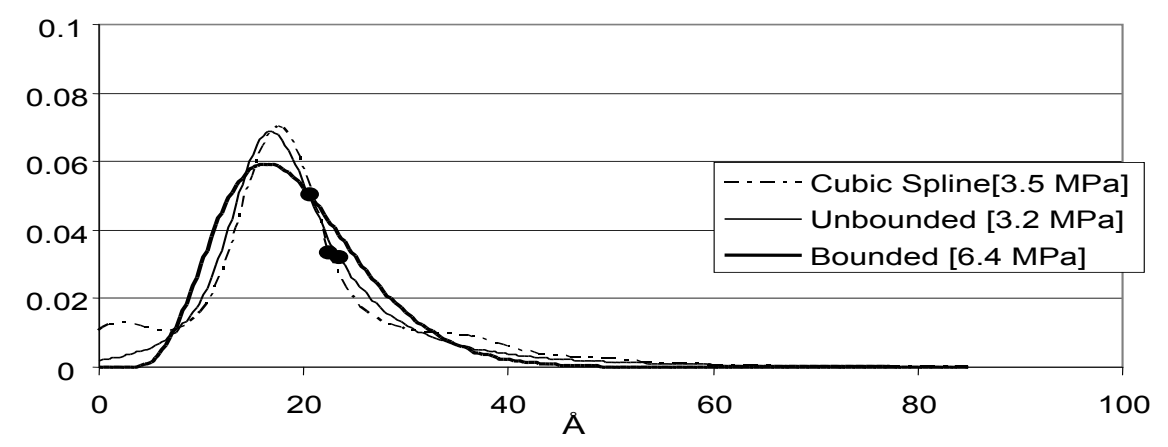

Figure 7: $\mathrm{Na}^{+}$Cubic Configuration with neglected end effects. PDF curves for the distribution of $r$-values and corresponding root mean square value, $r_{\mathrm{o}}$, for cubic spline, Johnson bounded, and Johnson unbounded distributions.

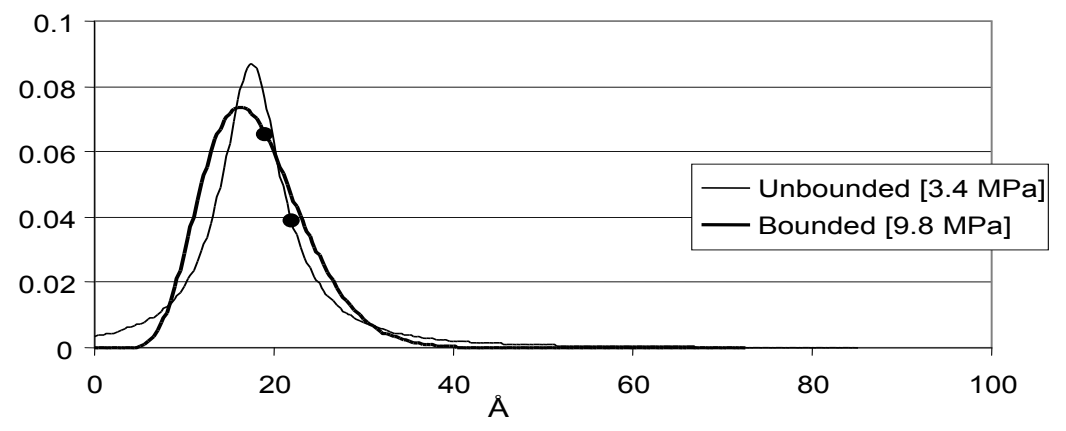

Figure 8: $\mathrm{Na}^{+} \mathrm{HCP}$ Configuration with neglected end effects. PDF curves for the distribution of $r$ values and corresponding root mean square value, $r_{\mathrm{o}}$, for Johnson bounded and Johnson unbounded distributions.

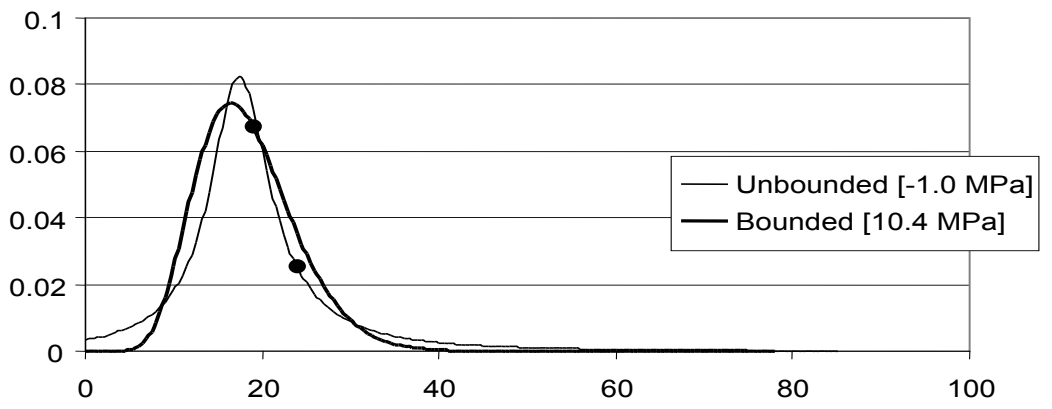

Figure 9: $\mathrm{Li}^{+}$Cubic Configuration with neglected end effects. PDF curves for the distribution of $r$ values and corresponding root mean square value, $r_{\mathrm{o}}$, for Johnson bounded and Johnson unbounded distributions. 


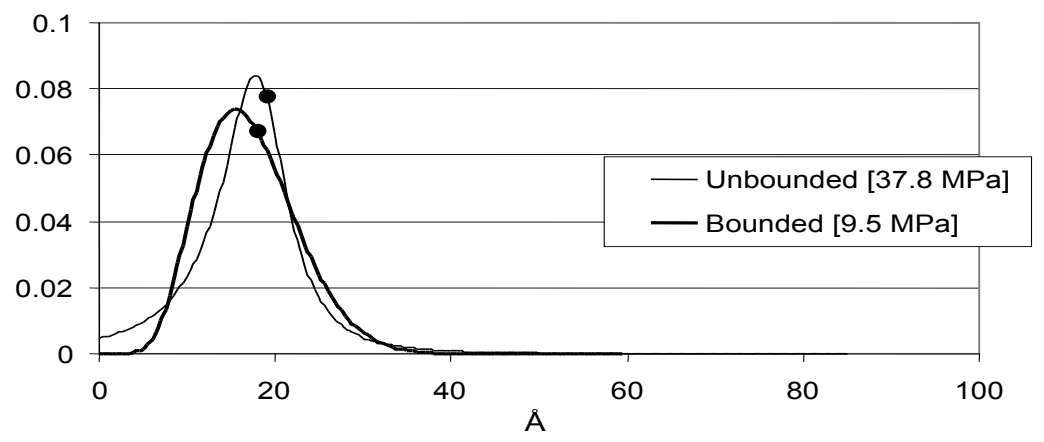

Figure 10: $\mathrm{Li}^{+} \mathrm{HCP}$ Configuration with neglected end effects. PDF curves for the distribution of $r$ values and corresponding root mean square value, $r_{\mathrm{o}}$, for Johnson bounded and Johnson unbounded distributions.

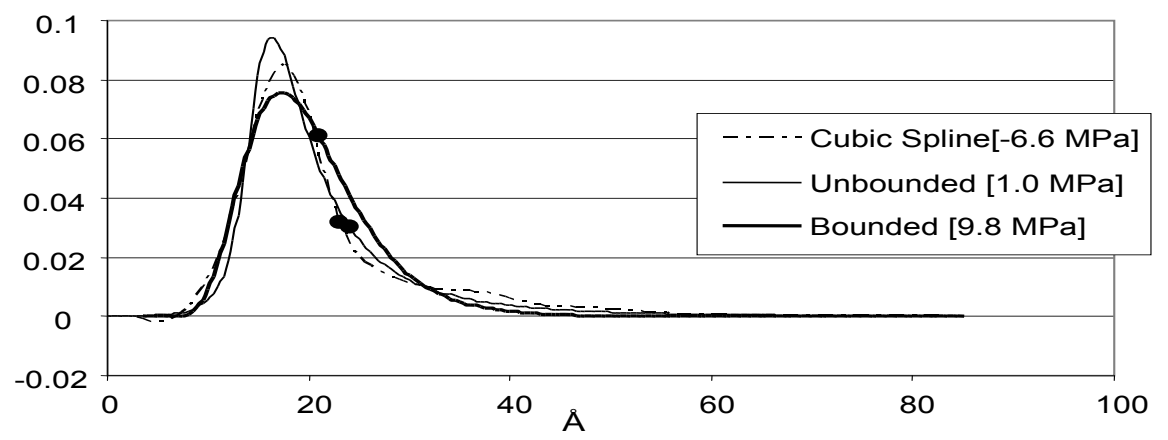

Figure 11: $\mathrm{Na}^{+}$Cubic Configuration with corrected end effects. PDF curves for the distribution of $r$-values and corresponding root mean square value, $r_{\mathrm{o}}$, for cubic spline, Johnson bounded, and Johnson unbounded distribution.

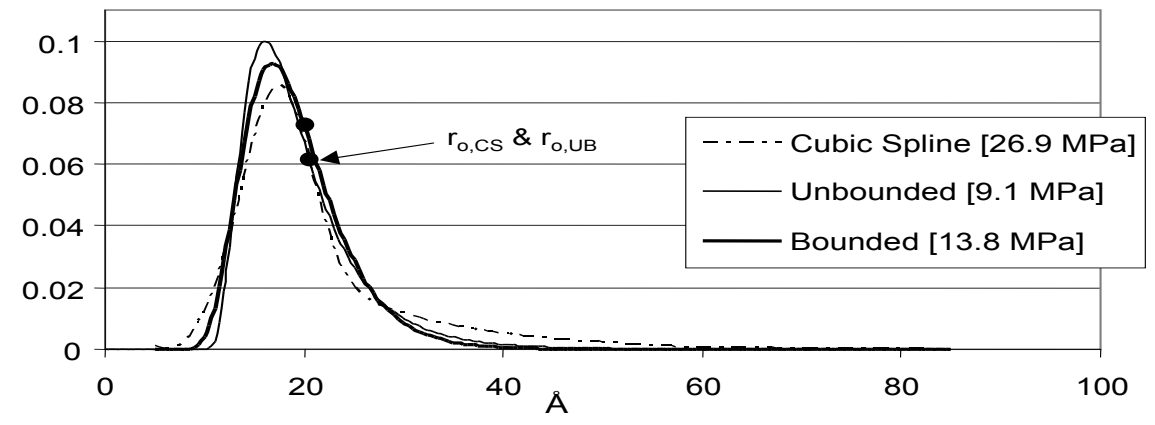

Figure 12: $\mathrm{Na}^{+} \mathrm{HCP}$ Configuration with corrected end effects. PDF curves for the distribution of $r$-values and corresponding root mean square value, $r_{\mathrm{o}}$, for cubic spline, Johnson bounded, and Johnson unbounded distributions. 


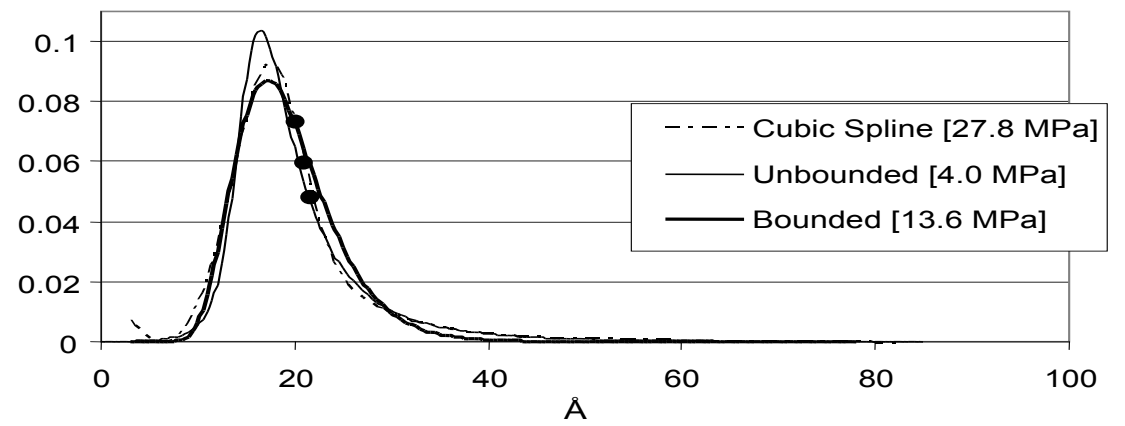

Figure 13: $\mathrm{Li}^{+}$Cubic Configuration with corrected end effects. PDF curves for the distribution of $r$-values and corresponding root mean square value, $r_{\mathrm{o}}$, for cubic spline, Johnson bounded, and Johnson unbounded distributions.

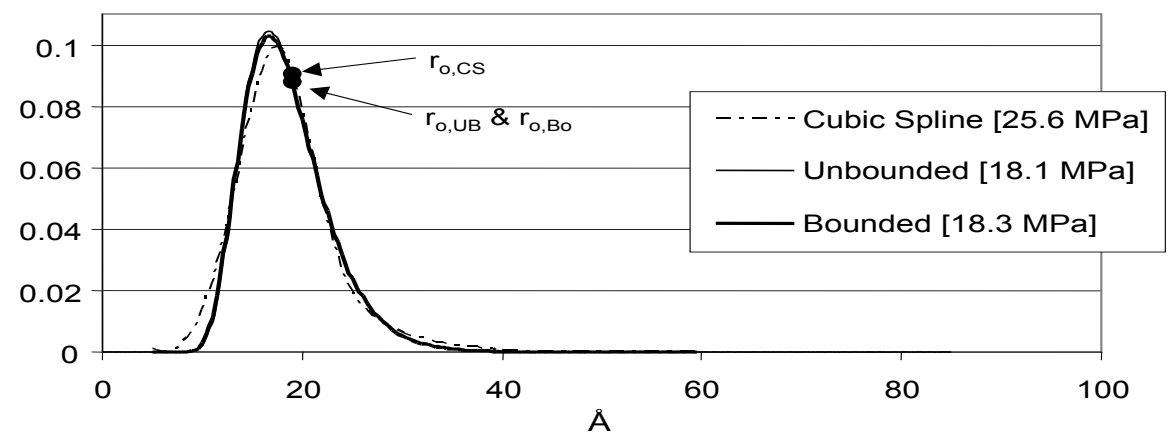

Figure 14: $\mathrm{Li}^{+} \mathrm{HCP}$ Configuration with corrected end effects. PDF curves for the distribution of $r$-values and corresponding root mean square value, $r_{\mathrm{o}}$, for cubic spline, Johnson bounded, and Johnson unbounded distributions.

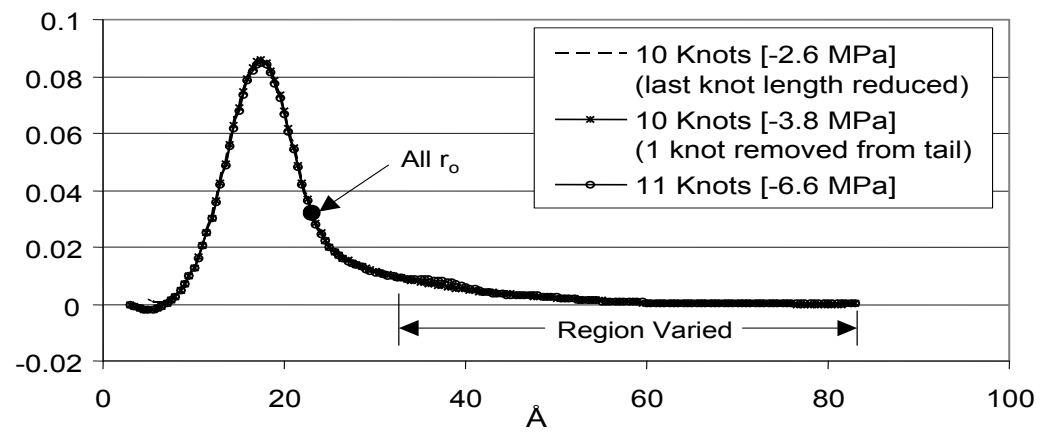

Figure 15: $\mathrm{Na}^{+}$Cubic Configuration with corrected end effects. PDF curves for the distribution of $r$-values and corresponding root mean square value, $r_{\mathrm{o}}$, for minor variations in cubic spline distributions with 10 and 11 knots. PDFs are nearly indistinguishable while stiffness predictions vary significantly. 
The traditional cubic spline approach is deemed the most unreliable of the considered methods because of the ill-defined methodology for fixing the knots. Any perception of consistency in stiffness predictions (based on the last 3 entries for cubic spline approach in Table 3), is somewhat misguided as these results are to some degree 'manufactured' by moving the knots until an appropriate response is predicted. While this may be acceptable for well-studied cases, one of the primary goals of modeling is to predict those cases which are not easily studied experimentally. As demonstrated in Figure 15, by altering the placement and number of knots we were able to alter the predicted stiffness. In the illustrative example of 15 , the cubic spline approach not only yields non-physical predictions (negative stiffness) from a data set for which the Johnson distributions yield positive predictions, but also illustrates that minor variation to the number and placement of knots has nonnegligible impact on predicted stiffness. The single greatest challenge with cubic spline is discerning the level of detail which is physically appropriate. With the Johnson cases, no qualitative judgment of this sort is required.

It is proposed that the reason the Johnson bounded case offers the most stable predictions is because the $r_{\mathrm{o}}$ position is consistently nearer the peak of the distribution. At this point in the $P(r)$ curve, the expression

$$
r_{\mathrm{o}} P\left(r_{\mathrm{o}}\right) P^{\prime \prime}\left(r_{\mathrm{o}}\right)-r_{\mathrm{o}}\left[P^{\prime}\left(r_{\mathrm{o}}\right)\right]^{2}+P\left(r_{\mathrm{o}}\right) P^{\prime}\left(r_{\mathrm{o}}\right)<0,
$$

which is a necessary condition for reasonable stiffness predictions (based on Equation (8)), is more likely to be satisfied because $P^{\prime \prime}$ is more likely to be negative (while we are generally assured that $P>0$ and $\left.P^{\prime}<0\right)$. Further, the magnitude of $P^{\prime}$ is likely less sensitive to small changes in $r_{\mathrm{o}}$ in this region.

\section{Predicted Stress-Strain Responses}

Figure 16 illustrates the predicted stress-strain response for the bounded cases, with corrected end-effects and where the nominal stress has been normalized with respect to $E_{\text {init }}$ to facilitate direct comparison to experimental data. It is seen that up to several percent strain, the predicted stress-strain behavior is consistent with experiment [36]. Moreover, while emphasis is being placed 

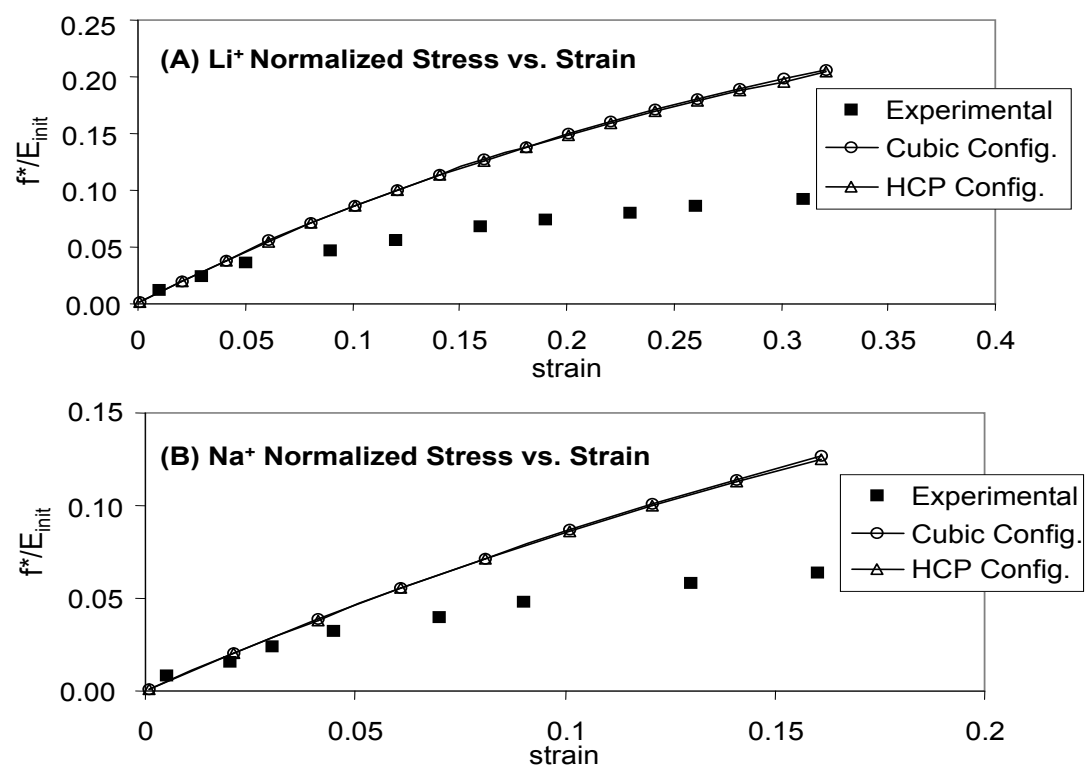

Figure 16: Normalized Stress-Strain behavior of experimental data [29] and model, both Cubic and HCP cluster configurations, with Corrected End Effects.

on the Johnson bounded with corrected end effects cases, it can be shown that within the range of several percent strain, all of the Johnson predictions (normalized with respect to $E_{\text {init }}$ ) yield similar stress-strain response trends. The cubic spline cases introduce considerably more variability and, in general, yield normalized stress predictions that are considerably higher than the Johnson cases at a given strain.

Further, it is not appropriate to compare model and experiment predictions beyond several percent strain because beyond this point the strain response is known to be effected by the hydration technique. Therefore, within the context of the traditional use of RIS (viz., normalized comparisons only), the modeling methodology is deemed appropriate and useful for study of solvated Nafion, both in terms of treatment of cluster communication concept for identification of load bearing chain lengths and the statistical analysis.

Traditionally, nominal stress and modulus are normalized with respect to $\nu k T$, from which physical trends may be inferred. Figures 16 and 17 consider this approach, where stress-strain end points correspond to failure points (known experimentally). Figure 17 applies this approach to illustrate that correction of the end effects leads to stiffer predicted responses throughout the stress-strain curve. Similarly, Figure 18 illustrates that assuming an HCP cluster morphology yields stiffer stress-strain response than assuming a Cubic morphology. Therefore, because the 

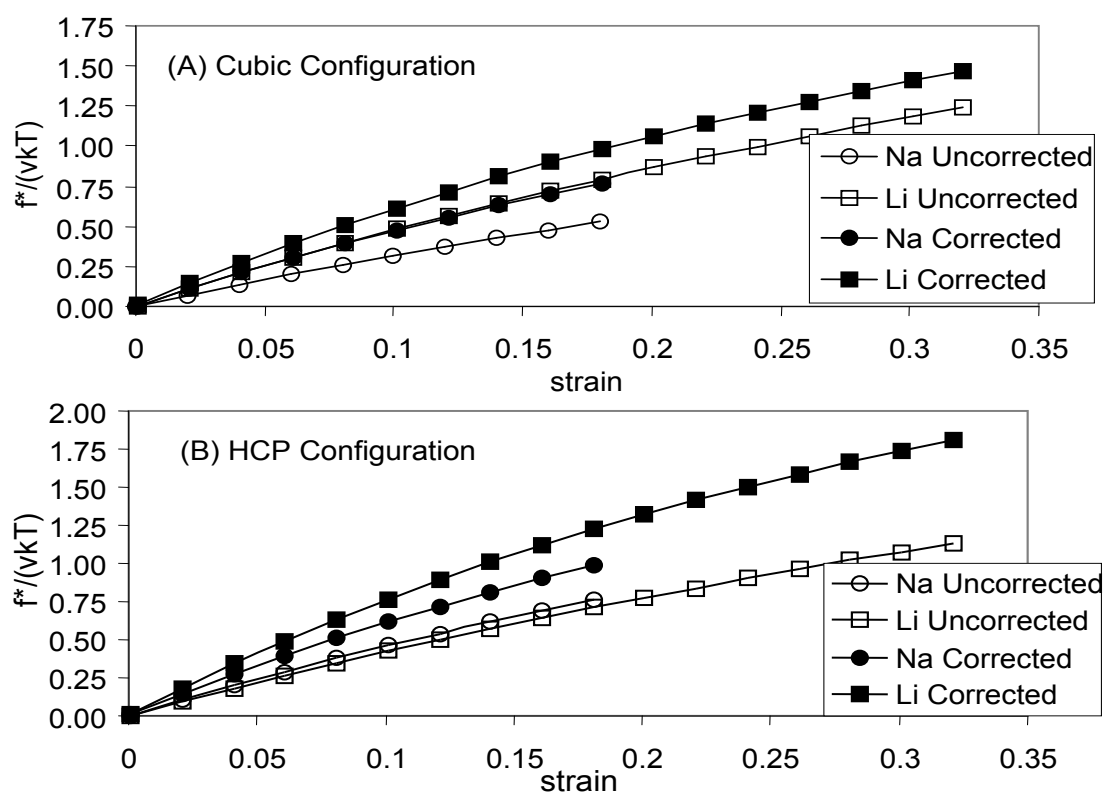

Figure 17: Stress normalized with respect to $\nu k T$ versus strain for bounded distributions, with neglected and corrected end effects.

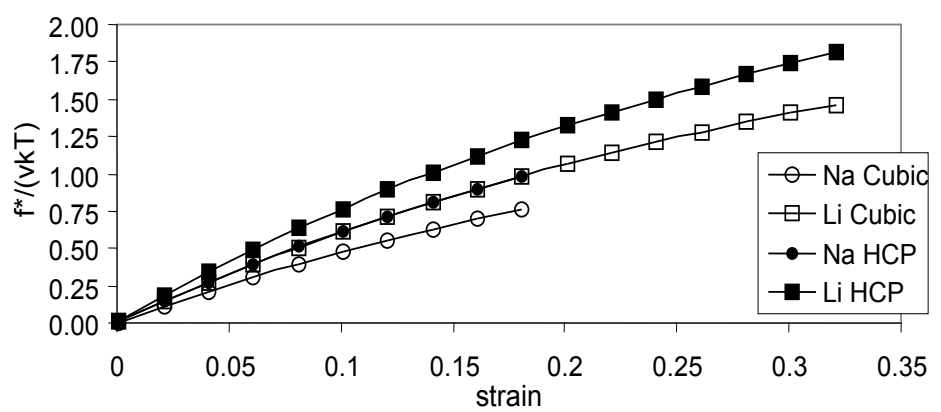

Figure 18: Stress normalized with respect to $\nu k T$ versus strain, corrected end effects with bounded distributions, for Cubic and HCP configurations.

cluster diameter and volume fractions are held constant for the two cases, it must be concluded that cluster morphology plays an important role in material stiffness.

Similarly, the Johnson bounded cases tend to predict higher stiffness than the Johnson unbounded cases (for normalization with respect to $\nu k T$ ). It can be shown that this trend persists throughout the stress-strain response as a whole. Moreover, the stable nature of the stiffness predictions for the bounded cases is mirrored in stable stress-strain trends as illustrated above, while the unbounded and cubic spline cases are much more likely to have erratic stress-strain response (mirroring the erratic stiffness predictions). 

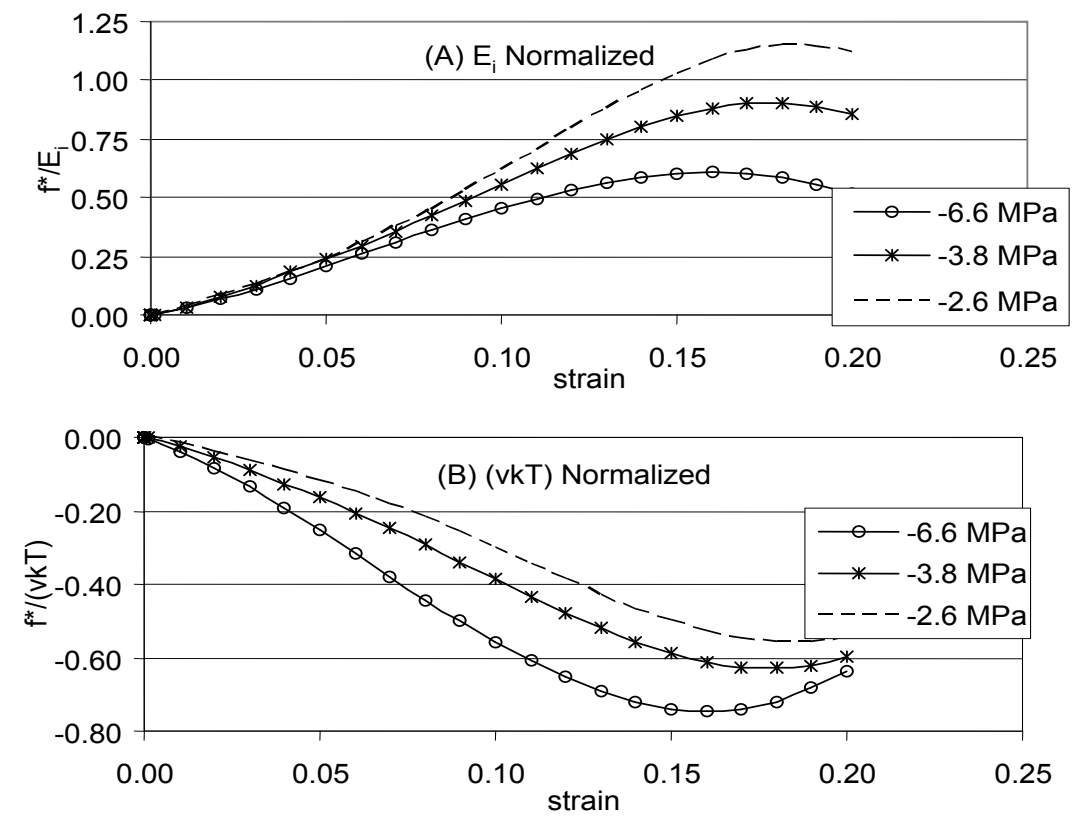

Figure 19: $\mathrm{Na}^{+}$Cubic configuration with corrected end effects. Stress-strain predictions normalized with respect to $E_{i}$ and $v k T$ for minor variations in cubic spline distributions (refer to Figure 15).

Figure 19 revisits the cubic spline cases of Figure 14 to illustrate the corresponding predicted stress-strain response trends. Recalling from Figure 15 that subtle changes to $P(r)$ via knot selection lead to non-negligible variation in the stiffness predictions, it is observed that the same continues to be true for stress-strain response trends. In particular, the variations in response illustrated in Figure 19, where the predictions are normalized with respect to $\nu k T$, is significant as this normalization has often been used to infer physical meanings between similar distribution data (i.e., increasing volume fractions of rigid inclusions [26]). It is of course presumed that previous practitioners of this modeling methodology have been aware of this sensitivity when present in their own systems and were thus potentially subjected to a substantial task in identifying the proper cubic spline fit. It is thus again emphasized that application of the Johnson bounded case represents both stability and simplification in RIS predictions as compared to the cubic spline case.

\section{Johnson Parameter Perturbation Results}

Perturbation studies yield insight into the physical significance of the various Johnson parameters. Table 4 provides sample results of the perturbation studies (for the case of Lithium, HCP, corrected end-effects, Johnson bounded). In general, it is found that variation of $\xi$ (location parameter) has 
the most significant impact; variation of $\delta$ (shape/peak parameter) and $\lambda$ (scale/spread parameter) have weaker but discernable effects; and variation of $\gamma$ (shape/skewness parameter) has a variable effect.

Table 4: Perturbation study of Lithium, HCP, Johnson Bounded, corrected end effects.

\begin{tabular}{cccccc}
\hline \hline$\xi$ & $\delta$ & $\lambda$ & $\gamma$ & $r_{\mathrm{o}}(\AA)$ & $E_{i}(\mathrm{MPa})$ \\
\hline 4.17 & 2.977 & 248.7 & 8.689 & 18.055 & 16.4 \\
5.17 & 2.977 & 248.7 & 8.689 & 19.0275 & 18.1 \\
6.17 & 2.977 & 248.7 & 8.689 & 20.0027 & 21.1 \\
\hline 5.17 & 2.777 & 248.7 & 8.689 & 16.6582 & 17.0 \\
5.17 & 2.977 & 248.7 & 8.689 & 19.0275 & 18.1 \\
5.17 & 3.177 & 248.7 & 8.689 & 21.4726 & 19.6 \\
\hline 5.17 & 2.977 & 244.7 & 8.689 & 18.8024 & 18.3 \\
5.17 & 2.977 & 248.7 & 8.689 & 19.0275 & 18.1 \\
5.17 & 2.977 & 252.7 & 8.689 & 19.2525 & 18.0 \\
\hline 5.17 & 2.977 & 248.7 & 7.689 & 24.1299 & 16.4 \\
5.17 & 2.977 & 248.7 & 8.689 & 19.0275 & 18.1 \\
5.17 & 2.977 & 248.7 & 9.689 & 15.2235 & 19.9 \\
\hline
\end{tabular}

Increasing $\xi$ always leads to an increase in the predicted stiffness. To interpret the physical significance of this, the analysis must be considered within the context of Equation (19). From this equation it is seen that the chain density $\nu$ is held constant while effectively increasing the typical chain length. In order for this to happen both the material density $\rho$ and molecular weight must rise, meaning that any given bond within the higher $\xi$ material is carrying less load for a given external load. This corresponds to an increase in the load carrying capability for the bulk material, or higher stiffness.

Increasing $\delta$ or decreasing $\lambda$ have similar effects on the distribution, where both lead to a more peaked distribution and marginally higher predicted stiffness. To say that a distribution is more peaked also suggests that the molecular weight distribution is narrower. It has been found that a narrower molecular weight distribution corresponds to a stiffer material in other polymers such as HDPE, so it is within reason that the same would be true for this class of polymers. 


\section{Conclusions}

A new multiscale modeling technique for the prediction of ionomer stiffness has been presented through extension of traditional RIS theory. Prediction stability is introduced by fitting the chain length distribution $P(r)$ using a Johnson bounded distribution rather than the commonly applied cubic spline approach. For all considered cases, the Johnson bounded cases yielded the most stable stiffness and stress-strain predictions, and the cubic spline approach yielded the most unreliable (variable) results. A Johnson distribution is further preferred because its parameters can be related to physical characteristics in the polymer, whereas cubic spline parameters cannot. Further, the normalized stress-strain response measured experimentally is consistent with that predicted by the model up to several percent strain (when appropriately normalized). Direct prediction of stiffness and stress-strain response may ultimately be possible once sources of error such as early termination and estimation of the molecular weight are addressed in detail. Lastly, the current model gives insight into significant material parameters. For example, correction of end effects yields higher predicted stiffness; thus end effects are non-negligible in this relatively short chained system. Further, the stiffness predictions are sensitive to the assumed cluster morphology, offering a tool for studying this unresolved physical issue.

\section{Acknowledgements}

The authors would like to thank Garth Wilkes (Virginia Tech) for multiple enlightening discussions on RIS theory, Jessica Mathews for her contributions to the construction of the preliminary computational code, and Jim Wilson (NCSU) for input regarding the density fitting techniques . Collaboration was facilitated while the authors were visitors at the Statistical and Applied Mathematical Sciences Institute (SAMSI), Research Triangle Park, NC, and the research of EKL was supported in part by the Science Foundation through the SAMSI grant DMS-0112069. LMW and DJL were supported by National Science Foundation Grant CMS 0093889 whereas RCS was supported in part by the Air Force Office of Scientific Research through the grant AFOSR FA9550-04-1-0203. The authors gratefully acknowledge this support. 


\section{References}

[1] K. Sadeghipour, R. Salomon, and S. Neogi: Development of a novel electrochemically active membrane and 'smart' material based vibration sensor/damper. Smart Mater. Struc. 1(2), 172 (1992).

[2] K. Oguro, Y. Kawami, and H. Takenaka: An actuator element of polyelectrolyte gel membraneelectrode composite. Osaka Kogyo Gijutsu Shikensho Kiho 43(1), 21 (1992).

[3] Yoshito Osada and Mariko Hasebe: Electrically Activated Mechanochemical Devices Using Polyelectrolyte Gels. Chem. Lett. 1285 (1985).

[4] Masahiro Irie: Photoresponsive Polymers. Reversible Bending of Rod-Shaped Acrylamide Gels in an Electric Field. Macromolecules 19, 2890 (1986).

[5] Tohru Shiga and Toshio Kurauchi: Deformation of Polyelectrolyte Gels Under the Influence of Electric Filed. Journal of Applied Polymer Science 39(11/12), 2305 (1990).

[6] M. Shahinpoor: Conceptual Design, Kinematics and Dynamics of Swimming Robotic Structures Using Ionic Polymeric Gel Muscles. Smart Materials and Structures 1(1), 91 (1992).

[7] M. Shahinpoor, Y Bar-Cohen, J.O. Simpson, and J. Smith: Ionic polymer-metal composites (IPMCs) as biomimetic sensors, actuators and artificial muscles - a review. Smart Mater. Struct. 7(6), R15 (1998).

[8] Y. Bar-Cohen (Editor), Electroactive Polymer (EAP) Actuators as Artificial Muscles - Reality, Potential, and Challenges (SPIE- The International Society for Optical Engineering, Bellingham, Washington, 2001).

[9] M. Mojarrad and M. Shahinpoor: Ion exchange membrane-platinum composites as electrically controllable artificial muscles. SPIE Proc 2779 p. 1012 (1996).

[10] S. Nemat-Nasser: Micromechanics of Actuation of Ionic Polymer-metal Composites (IPMCs). J. Appl. Phys. 92(5), 2899 (2002).

[11] K. Farinholt, K. Newbury, M. Bennet, and D. Leo: An Investigation into the Relationship between Charge and Strain in Ionic Polymer Sensors, in First World Congress on Biomimetics and Artificial Muscles (Albuquerque, NM, 9 -11 December 2002).

[12] W.Y. Hsu and T.D. Gierke: Elastic Theory for Ionic Clustering in Perfluorinated ionomers. 
Macromolecules 15, 101 (1982).

[13] V. K. Datye, P.L. Taylor, and A. J. Hopfinger: Simple Model for Clustering and Ionic Transport in Ionomer Membranes. Macromolecules 17, 1704 (1984).

[14] V. K. Datye and P. L. Taylor: Electrostatic Contributions to the Free Energy of Clustering of an Ionomer. Macromolecules 18, 1479 (1985).

[15] A. Lehmani, S. Durand-Vidal, and P. Turq: Surface Morphology of Nafion 117 Membrane by Tapping Mode Atomic Force Microscope. J. Appl. Poly. Sci. 68, 503 (1998).

[16] V. Barbi, S. Funari, R. Gehrke, N. Scharnagl, and N. Stribeck: Nanostructure of Nafion membrane material as a function of mechanical load studied by SAXS. Polymer 44, 4853 (2003).

[17] P.G. de Gennes, Ko Okumura, M. Shahinpoor, and K.J. Kim: Mechanoelectric Effects in Ionic Gels. Europhysics Letters 50(4), 513 (2000).

[18] K. Asaka and K. Oguro: Bending of polyelectrolyte membrane platinum composites by electric stimuli. J. Elec. Chem. 480, 186 (2000).

[19] J. Y. Li and S. Nemat Nasser: Micromechanical analysis of ionic clustering in Nafion perfluorinated membrane. Mech. Mater. 32, 303 (2000).

[20] S. Nemat-Nasser and J.Y. Li: Electromechanical response of ionic polymer-metal composites. J. Appl. Phys. 87(7), 3321 (2000).

[21] L.M. Weiland and D.J. Leo: Computational Analysis of Ionic Polymer Cluster Energetics. J. Appl. Phys. 97, 013541 (2004).

[22] L.R.G. Treloar, The Physics of Rubber Elasticity, 3rd ed. (Clarendon Press, Oxford, England, 1975).

[23] W.G.F. Grot, C.E. Munn, P.N. Walmsley, in 141st Meeting of the Electrochemical Society, Houston, TX, May 1972.

[24] P.J. Flory, Statistical Mechanics of Chain Molecules (Hanser Publishers, New York, New York, 1988).

[25] J.E. Mark and J.G. Curro: A non-Gaussian theory of rubberlike elasticity based on rotational iomeric state simulations of network chain configurations. I. Polyethylene and Polydimethyl- 
siloxane short-chain unimodal networks. J. Chem. Phys. 79(11), 5705 (1983).

[26] Q.W. Yuan, A. Kloczkowski, J.E. Mark, and M.A. Sharaf: Simulations on the Reinforcement of Poly(dimethylsiloxane) Elastomers by Randomly Distributed Filler Particles. J. Poly. Sci B: Poly. Phys 34, 1647 (1996).

[27] M.A. Sharaf, A.Kloczkowski, and J.E. Mark: Monte Carlo simulations on reinforcement of an elastomer by oriented prolate particles. Comp. Theo. Poly. Sci. 11, 251 (2001).

[28] M.A. Sharaf, R.H. Abouhussein: Computer Simulations on the chain deformations of Poly(Ethylene) by Randomly-Oriented Prolate Filler particles Arranged on a Cubic Lattice. Poly. Preprints 44(1), 1258 (2003).

[29] M.A. Sharaf, I.M. Jasiuk, K.I. Jacob: Monte Carlo Simulations combined with Micromechanics to predict effective elastic moduli of elastomeric nanocomposites filled with unidirectional rigid inclusions. Poly. Preprints 44(1), 1249 (2003).

[30] T.W. Bates and W.H. Stockmayer: Conformational Energies of Perflouroalkanes. II Dipole Moments of H(CF2)nH. Macromolecules 1(1), 12 (1968).

[31] J.L. Mathews, E.K. Lada, L.M. Weiland, R.C. Smith, D.J. Leo: Monte Carlo Simulation of a Solvated Ionic Polymer with Cluster Morphology. Submitted to Smart Materials and Structures January 2005.

[32] K.A. Page and R.B. Moore: Correlations between bulk mechanical relaxations and spin diffusion times in Perfluorosulfonate Ionomers: Molecular Origins of Mechanical Relaxations. Polymer Preprints 44(1), 1144 (2003).

[33] A.K. Phillips and R.B. Moore: Phase Behavior in Solution Processed Perfluorosulfonate Ionomers. Polymer Preprints 44(1), 1142 (2003).

[34] R.M. Jones, Mechanics of Composite Materials (McGraw-Hill Book Company, New York, New York, 1975).

[35] D.J. DeBrota, R.S. Dittus, S.D. Roberts, J.R. Wilson, J.J. Swain, and S. Venkatraman: Modeling Input Processes with Johnson Distributions. Proc. of the Winter Simulation Conf. p. 308 (Piscataway, New Jersey: IEEE, 1989).

[36] Y. Kawano, Y. Wang, R.A. Palmer, and S.R. Aubuchon: Stress-Strain Curves of Nafion 
Membranes in Acid and Salt Forms. Polimeros 12(2), 96, ISSN 0104-1428 (2002). 